\title{
Ambient Atoms : un périphérique pour la visualisation ambiante d'informations
}

\author{
Ambient Atoms: a Device for Ambient Information Visualization
}

\author{
Sébastien Crouzy ${ }^{1}$, Stan Borkowski ${ }^{2}$, Sabine Coquillart ${ }^{3}$ \\ ${ }^{1}$ INRIA, Université Grenoble Alpes, France, crouzy.sebastien@gmail.com \\ 2 INRIA, France, Stan.Borkowski@inria.fr \\ ${ }^{3}$ INRIA, Univ. Grenoble Alpes, CNRS, Lab LIG, France, Sabine.Coquillart@inria.fr
}

RÉSUMÉ. Les années récentes ont vu une explosion de la quantité d'informations disponibles. Cette explosion concerne aussi bien les informations publiques que privées. Au niveau individuel, cette abondance d'informations est grandement matérialisée par le système informatique qui ne nous quitte plus, à savoir, le smartphone. Le smartphone fournit un accès rapide à une multitude d'informations et de données d'une richesse incroyable. Toutes ces données sont presque toujours délivrées à l'utilisateur au moyen du même module de restitution: l'écran (épisodiquement le son ou les vibrations) du smartphone. Le smartphone est un moyen d'accès simple à différentes informations, mais ce n'est pas toujours le moyen le plus pratique, suivant le type de données, ou en fonction des circonstances d'utilisation. Les périphériques ambiants peuvent constituer une solution alternative à la visualisation d'informations sur le smartphone. L'affichage ambiant fournit un accès instantané et sans effort à l'information, d'un simple coup d'oeil.

Cet article propose un nouveau mode de visualisation ambiante, l'Ambient Atoms. L'Ambient Atoms est un objet connecté simple et modulable, qui se présente sous la forme d'un tableau sur lequel les informations sont visualisées de façon symbolique. Une application de visualisation d'informations relatives à un appartement est proposée comme exemple d'application.

ABSTRACT. Recent decades have seen an explosion of available information. This explosion concerns public as well as private information. From an individual perspective, the explosion is largely materialized by the computer system that we constantly have with us, namely the smartphone. The smartphone provides immediate access to a wealth of information and a multitude of data. All these various data are often delivered to the user by means of the same single output module ie. the screen (and episodically the sound or vibrations) of the smartphone. The smartphone is an easy way to get access to many different data, but this is not always the most practical solution for each category of information, nor in all circumstances. Ambient devices can be an answer to some situations for which the smartphone is not convenient enough to get access to the required information. Ambient displays deliver effortless, instant access to information at a glance. This paper introduces the Ambient Atoms, a new ambient visualization device. The Ambient Atoms is a cheep and flexible connected object that looks like a frame on which information is symbolically visualized. An application for the visualization of data relative to an apartment is proposed as a sample application.

MOTS-CLÉS. Internet des objets, visualisation ambiante, appartement intelligent.

KEYWORDS. Internet of Things, Ambient Visualization, Smart Home.

\section{Introduction}

Les années récentes ont vu une explosion de la quantité d'informations disponibles. Cette explosion concerne aussi bien les informations publiques que privées. A titre d'exemple, citons les prévisions météorologiques, ou les informations de trafic. Les informations privées sont elles largement issues de la multitude de capteurs qui commencent à scruter notre environnement et nos faits et gestes. Au niveau individuel, cette abondance d'informations est grandement matérialisée par le système informatique qui ne nous quitte plus, à savoir, le smartphone. Le smartphone fournit un accès rapide à une multitude d'informations et de données d'une richesse incroyable. Toutes ces données sont presque toujours délivrées à l'utilisateur au moyen du même module de restitution : l'écran (épisodiquement le son ou les vibrations) du smartphone. Le smartphone est un moyen d'accès simple à différentes informations, mais ce n'est pas toujours le moyen le plus pratique, suivant le type de données, ou en fonction des circonstances d'utilisation. En général, l'utilisation du smartphone nécessite de suspendre son activité pour tout d'abord, sortir son téléphone de sa poche ou de son sac, puis de lancer 
l'application souhaitée, ou d'interroger le web. De surcroît, si l'activité courante salit ou mouille les mains, le dérangement est encore plus important. Les notifications répondent partiellement au problème, mais elles ne résolvent pas tous les cas. De surcroît, la multiplication des notifications devient vite lourde et difficilement gérable.

Les périphériques ambiants peuvent constituer une solution alternative à la visualisation d'informations sur le smartphone. L'affichage ambiant fournit un accès instantané et sans effort à l'information, et ce, d'un simple coup d'oeil.

Ce papier propose un nouveau mode de visualisation ambiante, l'Ambient Atoms. L'Ambient Atoms est un objet connecté simple et modulable, qui se présente sous la forme d'un tableau sur lequel les informations sont visualisées de façon symbolique. Une application de visualisation d'informations

relatives à un appartement est proposée comme exemple d'application. On peut cependant imaginer de très nombreuses autres applications dans la vie de tous les jours, telles que visualiser le besoin des plantes en eau ou/et fertilisant, informer d'une date de péremption proche de denrées alimentaires, visualiser les prévisions météorologiques dans plusieurs régions d'intérêt, afficher les retards ou annulations des transports en commun empruntés quotidiennement, afficher l'agenda des proches de la famille, afficher les résultats des matches des championnats de son sport favori, afficher les valeurs en bourse de ses sociétés préférées, prévenir d'appels téléphoniques, de mails, ou de SMS envoyés par les proches,...

La suite de cet article est structurée comme suit : le deuxième paragraphe défini et présente un bref état de l'art des systèmes de visualisation ambiants, le troisième paragraphe introduit l'Ambient Atoms d'un point de vue utilisateur, le paragraphe suivant présente les composants matériels et logicielles constituant l'Ambient Atoms. Enfin, le cinquième paragraphe décrit un premier prototype développé pour la visualisation de diverses données relatives à un logement telles que la température de chaque

pièce, la présence de personnes, l'ouverture, ou non, des portes et fenêtres, les consommations électriques et de gaz.

\section{Travaux antérieurs}

Les premières recherches sur les affichages ambiants remontent à la fin des années 90 . L'objectif des affichages ambiants est de permettre aux utilisateurs de garder un œil sur des informations utiles mais non centrales à leur activité, ceci d'une manière simple et discrète, à l'aide d'un système d'affichage intégré dans l'environnement. Pousman et Stasko [1], proposent un ensemble de propriétés pour caractériser les systèmes d'information ambiants. Ils décrivent des systèmes d'affichage :

- d'informations importantes mais non critiques,

- qui peuvent passer de la périphérie au centre de l'attention des usagers, et vice versa,

- dont la représentation est tangible dans l'environnement,

- dont les mises à jour sont discrètes, et ne doivent pas déranger les usagers,

- plaisants esthétiquement, et approprié à l'environnement.

De nombreux périphériques d'affichage ambiant ont été proposés. Nous nous limiterons ici aux périphériques visuels. Parmi ceux-ci, on peut distinguer les systèmes à base d'écrans tels que CareNet [2], Infocanvas[3], Kandinsky [4] Chumby [5], Digital Family Portraits [6], et ceux, qui n'intègrent pas d'écran.

Nous allons nous intéresser ici plus particulièrement aux affichages ambiants n'intégrant pas d'écran. 


\subsection{Affichages ambiants dédiés, n'intégrant pas d'écran}

Parmi les affichages ambiants n'intégrant pas d'écrans, la plupart sont dédiés à une application donnée, comme : le LockDoll [7] qui est une petite figurine qui, par ses mouvements, transmet de façon discrète les informations d'un smartphone à son propriétaire ; le Dangling String [8], qui visualise la charge d'un réseau informatique ; ou le Lumitouch [9] qui est un ensemble de deux cadres avec photos qui permettent de transmettre des émotions à une personne à distance.

Les affichages ambiants dédiés à une application sont aussi souvent la transformation d'un objet de la vie courante tel qu'un parapluie avec l'Ambient Umbrella [10], ou un cordon d'alimentation électrique, avec le Power-Aware Cord [11]. Dans certains cas, l'affichage ambiant s'appuie même sur un élément naturel, comme le ciel, avec le Nuage vert [12].

\subsection{Affichages ambiants génériques, n'intégrant pas d'écran}

Un des plus connus, et des plus simple affichage ambiant n'intégrant pas d'écran, est l'Ambient Orb [13]. Il s'agit d'une boule dont la couleur change en fonction de la mise à jour de l'information. Limité à une seule information qui peut prendre plusieurs valeurs (couleurs), l'Ambient Orb est commercialisé par la société Ambiant Devices Inc. Une extension de l'Ambient Orb a été proposée par la société Moores Cloud. L'Holiday [14] est une guirlande de leds ou chaque led peut prendre une couleur différente, et peut être adressée indépendamment. Les Water Lamp et Pinwheels [15] sont deux autres exemples de systèmes d'affichage ambiant générique n'intégrant pas d'écran.

\section{Ambient Atoms - point de vue utilisateur}

Ces travaux se situent dans la suite directe des travaux sur l'Ambient Orb [13], l'Holiday de la société Moores Cloud [14] et l'Infocanvas [3]. L'Ambient Atoms est une version étendue, et surfacique de l'Ambient Orb ou de l'Holiday. On peut aussi le voir comme une version plus simple et plus modeste de l'Infocanvas, qui n'intègre pas d'écran. L'écran est ici remplacé par un mode d'affichage bas coût constitué d'un fond de tableau et de leds positionnées sur le tableau appelées atoms. Comme l'Ambient Orb, l'Holiday ou l'Infocanvas, l'Ambient Atoms est un affichage ambient générique. De ce fait il est adaptable à un grand nombre d'applications variées. Le fond de tableau va fournir toutes les informations pérennes, en particulier les informations qui expliquent le rôle et la signification de chaque atom, alors que les leds fournissent les informations mises à jour en temps réel. Outre l'exemple de la visualisation des informations relatives à un appartement connecté qui est présenté au paragraphe 5, citons un ou deux autres exemples d'applications possibles. La figure 1 présente un Ambient Atoms prévenant des appels téléphoniques, des sms, ou des messages électroniques provenant de proches.

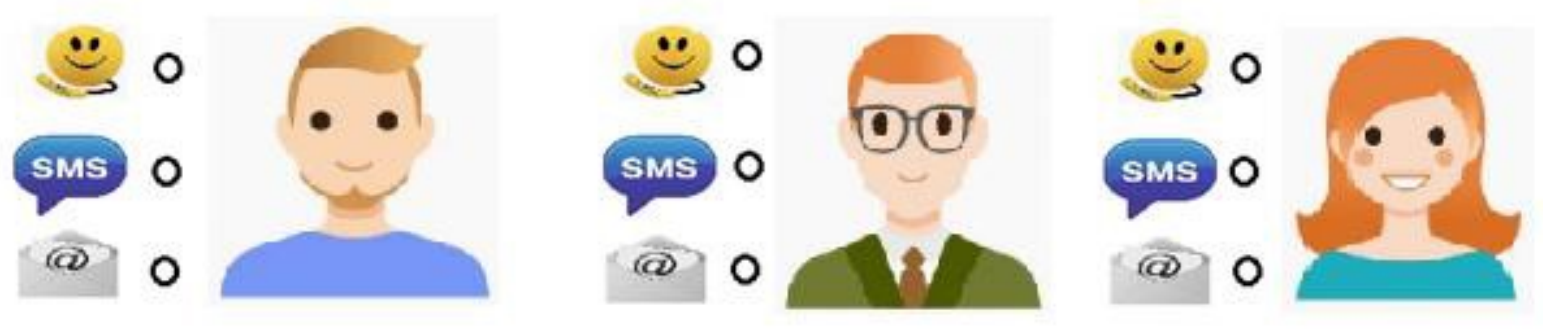

Figure 1. Appels des proches

De même, la figure 2 propose un Ambient Atoms simple employé pour visualiser l'agenda des personnes de la famille. Pour chaque personne, figure sa présence à la maison ou au travail pour le jour même et le lendemain. Les anneaux noirs représentent les atoms, matérialisés par les leds dont la couleur, symbolisant l'information, est mise à jour en temps réel. 


\begin{tabular}{|l|c|c|c|c|c|c|c|c|}
\hline Agenda & $\begin{array}{l}\text { AM } \\
\text { home }\end{array}$ & $\begin{array}{l}\text { PM } \\
\text { home }\end{array}$ & $\begin{array}{l}\text { AM } \\
\text { office/ } \\
\text { school }\end{array}$ & $\begin{array}{l}\text { PM } \\
\text { office/ } \\
\text { school }\end{array}$ & $\begin{array}{l}\text { Tomorrow } \\
\text { AM home }\end{array}$ & $\begin{array}{l}\text { Tomorrow } \\
\text { PM home }\end{array}$ & $\begin{array}{c}\text { Tomorrow } \\
\text { AM office }\end{array}$ & $\begin{array}{c}\text { Tomorrow } \\
\text { PM office }\end{array}$ \\
\hline Mick & $\mathbf{0}$ & $\mathbf{0}$ & $\mathbf{0}$ & $\mathbf{0}$ & $\mathbf{0}$ & $\mathbf{0}$ & $\mathbf{0}$ & $\mathbf{0}$ \\
\hline Jane & $\mathbf{0}$ & $\mathbf{0}$ & $\mathbf{0}$ & $\mathbf{0}$ & $\mathbf{0}$ & $\mathbf{0}$ & $\mathbf{0}$ & $\mathbf{0}$ \\
\hline John & $\mathbf{0}$ & $\mathbf{0}$ & $\mathbf{0}$ & $\mathbf{0}$ & $\mathbf{0}$ & $\mathbf{0}$ & $\mathbf{0}$ & $\mathbf{0}$ \\
\hline
\end{tabular}

Figure 2. Agenda de la famille

\section{Ambient Atoms - composants matériels et logiciels}

\subsection{Matériel - microcontrôleur}

Ce paragraphe décrit un premier prototype de l'Ambient Atoms développé dans la cadre de l'Equipex Amiqual4Home [16]. Les choix technologiques ne représentent qu'une approche parmi beaucoup d'autres approches tout aussi pertinentes pour mettre en œuvre l'Ambient Atoms. Ce paragraphe s'intéresse plus particulièrement à l'affichage des informations temps réel à base des leds. Le fond du tableau sera décrit sur l'exemple présenté au paragraphe suivant.

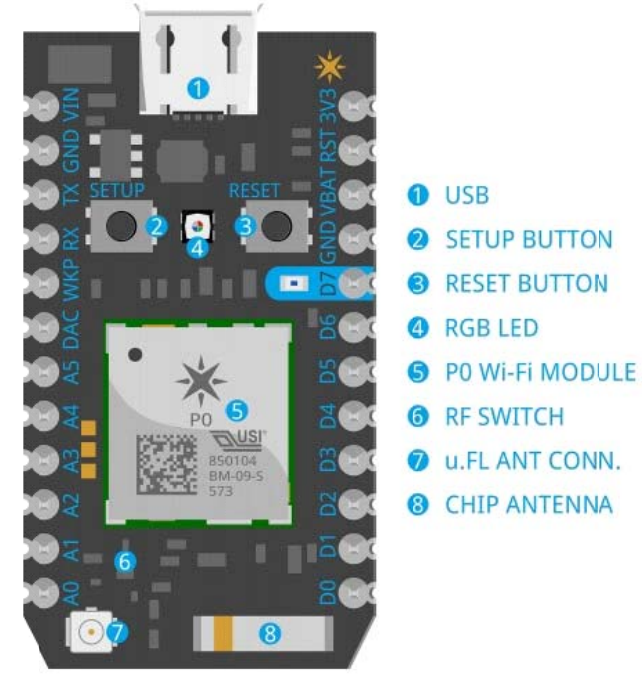

Figure 3. Le Particle's Photon (Source : https://www.particle.io/prototype)

Le premier prototype développé intègre le microcontrôleur Particle's Photon qui fait suite au Spark Core. Alimenté par USB, le microcontrôleur dispose d'une connection WiFi, et d'un MB de mémoire flash, et $128 \mathrm{~KB}$ de RAM pour exécuter le microprogramme. Tout périphérique déclaré et connecté au nuage (cloud) peut être géré en temps réel au travers du serveur web du Particle.io. Le microprogramme peut être développé en ligne en utilisant l'environnement de développement intégré (IDE) web du Particle. Le Particle utilise le même environnement de développement qu'Arduino et est donc compatible avec le code écrit pour l'Arduino avec peu ou pas de modifications. La programmation est réalisée en $\mathrm{C} / \mathrm{C}++$ ou en assembleur $\mathrm{ARM}$, et le code peut être compilé au niveau de l'IDE web. On peut aussi flasher le Particle directement à partir d'internet au travers de la connexion WiFi. Pour plus de détails, la figure 3 présente une photo du Particle's Photon, voir aussi [17]. 


\subsection{Matériel - LEDs}

Le premier prototype développé intègre une guirlande de leds WS2801 d'Adafruit, connectée au microcontrôleur, voir figure 4. L'état de chaque LED, représenté par sa couleur, peut être accédé et modifié indépendamment des autres LEDs. La couleur des LEDs fournit à l'utilisateur une représentation abstraite de l'information. Chaque LED intègre un contrôleur moulé dans le silicone. Les LEDs sont reliées par un câble à 4 conducteurs $(5 \mathrm{~V}$, terre, données, horloge). Les données sont transmises d'une LED à l'autre jusqu'à leur arrivée à la LED destinataire. La couleur de chaque LED est codée sur 24 bits représentant trois valeurs correspondant aux trois couleurs primaires (rouge, vert et bleu). Une valeur de 0 pour chacune des trois composantes correspond à une LED éteinte, alors qu'une valeur de 255 conduit à une LED blanche. L'information fournie par une LED est donnée par sa couleur. Elle peut aussi provenir de séquences d'animations variées, comme un clignotement. Dans ce but, plusieurs essais de séquences d'animation ont été testées.

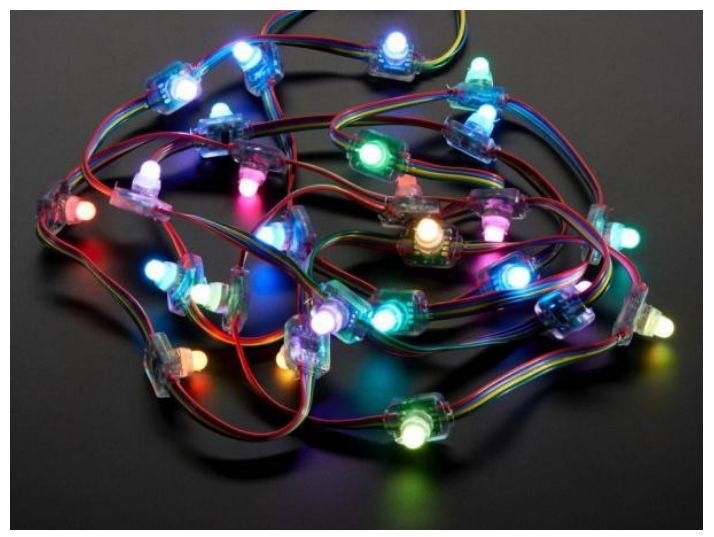

Figure 4. Adafruit WS2801 (Source : https://www.adafruit.com/products/738)

\subsection{Logiciel}

Le développement de l'Ambient Atoms utilise plusieurs bibliothèques pour : gérer un serveur web, traiter les chaînes JSON, et communiquer avec la guirlande de leds.

Le micrologiciel développé utilise les bibliothèques cJSON pour traiter les messages JSON issus des requêtes POST. Ces messages sont formatés sous forme d'une liste de LEDs avec leurs paramètres. Autant de LEDs que nécessaire peuvent être gérées indépendamment.

$\mathrm{Au}$ delà du changement de couleurs des LEDs, le micrologiciel intègre aussi plusieurs animations prédéfinies qui peuvent être activées à l'aide d'une requête POST sur le serveur web.

Les animations sont définies au moyen d'une structure contenant plusieurs paramètres nécessaires pour jouer l'animation. Parmi eux : le type d'animation (constant, clignotant, extinction progressive, aléatoire,...), la durée de l'animation, sa fréquence,...

\section{Ambient Atoms - prototype appliqué à la visualisation d'informations relatives aux logements}

Un premier prototype a été développé. Il permet la visualisation des informations relatives à un appartement connecté telles que l'ouverture/fermeture des portes et fenêtres, la consommation électrique, la consommation en eau, la présence de personnes dans les pièces,... Dans ce cas, le fond du tableau représente un plan de l'appartement, et chaque LED/atom fourni une information de type consommation, présence, ouverture/fermeture de porte ou fenêtre, voir figure 5. L'accrochage de l'Ambient Atoms sur le mur de la cuisine, par exemple, permet de superviser l'état de l'appartement d'un simple coup d'œil, de façon non intrusive. 


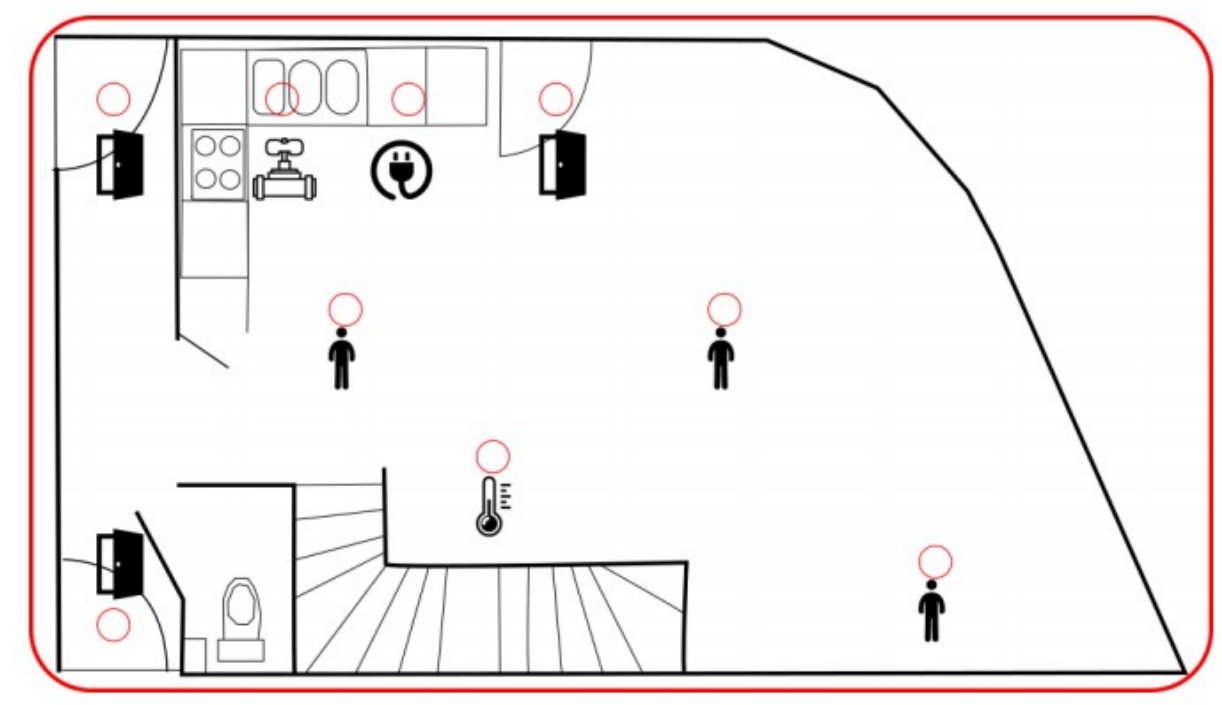

Figure 5. Schéma du prototype de l'Ambient Atoms dédié à la visualisation d'informations relatives à un appartement connecté

Le fond du tableau, qui varie en fonction de l'application, est ici une pièce de contreplaqué gravée avec un laser à commande numérique. Le plan de l'appartement, ainsi que des symboles permettant d'identifier les informations fournies par chaque LED sont gravés. Les trous permettant le positionnement des LEDs/atoms sont réalisés simultanément à la gravure, aussi avec un laser.

Neuf LEDs sont intégrées à l'Ambient Atom, elles servent à afficher la température, l'état des portes (ouvert/fermé), la présence de personnes dans les pièces, et la consommation électrique et de gaz.L'appartement est un appartement intelligent, disposant de nombreux capteurs. Les atoms représentent l'état des capteurs, matérialisé par la couleur ou/et l'animation des LEDs. A titre d'exemple, l'affichage de la température se fait au moyen du gradient de couleur classique allant du bleu au rouge, les consommations sont aussi visualisées de cette façon. L'ouverture des portes est visualisée par une couleur fixe, en l'occurrence, le vert, et l'information de présence est restituée par un clignotement des atoms. Le microcontrôleur et les câbles sont cachés derrière le fond du tableau. Voir figure 6 pour plus de détails. La photo est prise de l'entrée de la pièce, l'Ambient Atoms est posé sur le meuble de cuisine, à gauche de la photo. La porte que l'on aperçoit sur la gauche étant fermée, son atom, celui en haut à droite est par conséquent vert. De même, une personne étant présente, l'atom de présence situé au milieu en hauteur, et à droite est rouge (pour les besoins de l'article qui ne peut restituer les animations, un code vert/rouge à été choisi pour représenter la présence). Le capteur est fixé au plafond, au dessus de la table, à proximité de la lampe. La porte d'entrés (atom en bas à droite), et la porte d'accès au garage (atom en haut à gauche) étant toutes les deux fermées (elles ne sont pas visible sur la photo), les deux atoms associés sont verts. Les deux atoms en haut, entre les atoms d'ouverture des portes sont dédiés à la consommation électrique et à la consommation d'eau. Les deux atoms verts situés dans la pièce correspondent à deux autres atoms de présence, ils sont verts car personne n'est présent à proximité. Enfin, l'atom bleu (en bas au milieu), indique la température. 

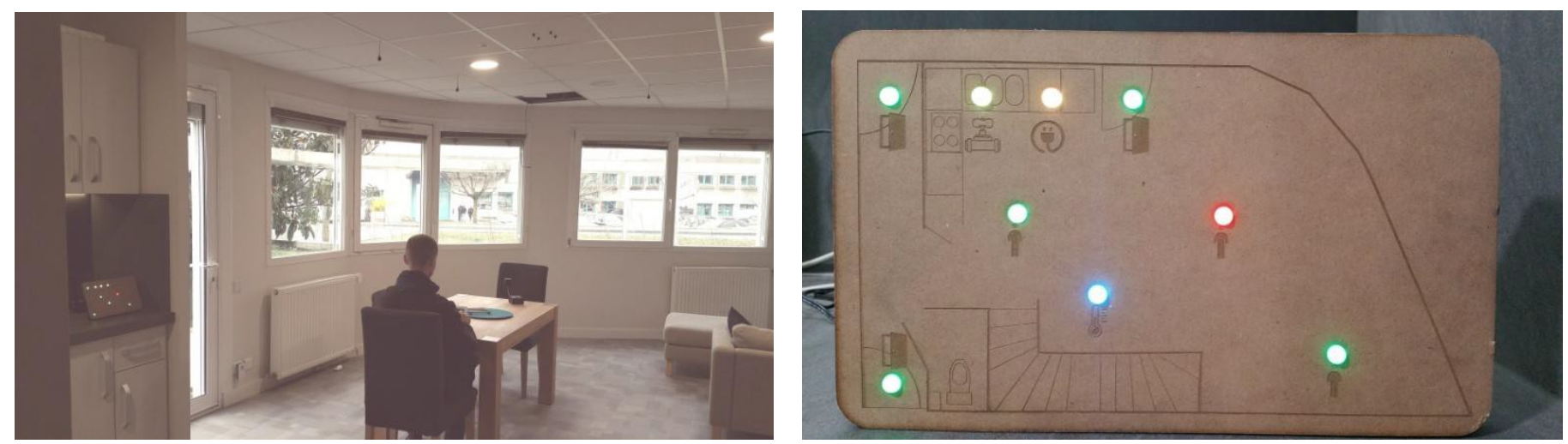

Figure 6. (a) Photo de l'appartement avec l'Ambient Atoms posé sur le meuble de cuisine, à gauche (b) Zoom sur l'Ambient Atoms. On notera la présence d'une personne symbolisée par une led rouge, et la porte fermée symbolisée par une led verte.

L'appartement intelligent ayant servi de test pour le premier prototype de l'Ambient Atoms est un appartement installé dans le cadre de l'Equipex Amiqual4Home [16], et géré par OpenHAB [18], un logiciel de domotique open source. L'appartement est totalement instrumenté de capteurs variés, il sert à tester des prototypes et de nouveaux services en grandeur nature. L'appartement intègre une multitude de capteurs de toutes sortes, allant des compteurs de consommation d'eau, d'électricité, de gaz, jusqu'à des capteurs de présence, de détection d'ouverture/fermeture des portes et fenêtres...Un plancher et un plafond démontables permettent l'ajout de capteurs à volonté.

OpenHAB permet de simplifier et fiabiliser l'ajout, le contrôle, la configuration, et la lecture de capteurs ou d'actionneurs. Il facilite aussi le traitement et le stockage des informations provenant des capteurs. OpenHAB permet aussi d'unifier la gestion de capteurs ou d'actionneurs utilisant des protocoles de communication différents grâce à la notion d'event bus. Chaque fois que l'état d'un périphérique change, une notification est envoyée sur le bus d'évènements. Voir figure 7.
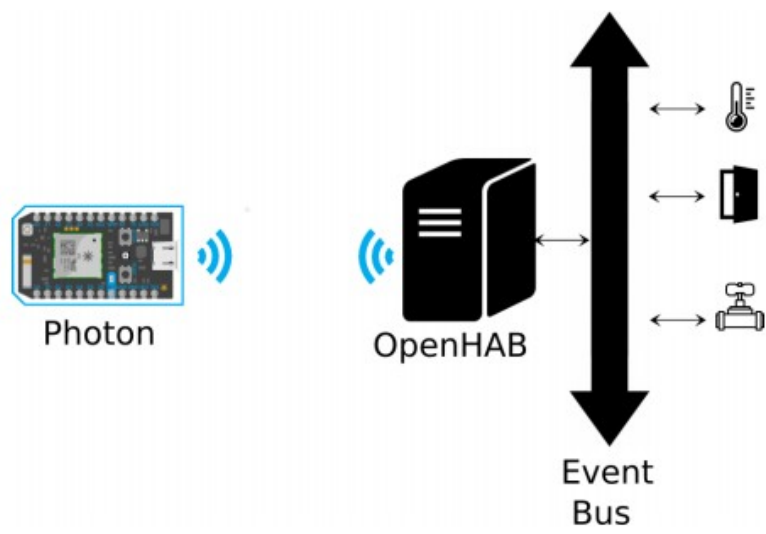

Figure 7. Communication entre le Photon, OpenHAB et les capteurs

OpenHAB Designer est le logiciel employé pour configurer les différents éléments et leurs connexions. Les commandes et les mises à jour du bus d'événements peuvent déclencher des règles définies dans OpenHAB Designer. Par exemple, un capteur de température peut être déclaré dans OpenHAB comme un élément de type Nombre. Cet élément est alors relié à son équivalent réel, dans ce cas un capteur de température. Chaque fois que l'état du capteur change, une notification est envoyée sur le bus d'événements, et des règles peuvent être écrites pour réagir à ce nouvel état. Nous pouvons, par exemple, définir une règle pour éclairer une LED sur un spectre allant du bleu au rouge pour visualiser la température émise par le capteur. 
Les règles sont également utilisées pour envoyer des commandes sur les éléments ou effectuer des opérations plus complexes. Ces opérations vont de l'envoi de requêtes HTTP à la mise en forme des valeurs de capteurs reçues, et leur transformation en données utilisables. Plusieurs règles pour envoyer des requêtes HTTP POST au photon afin de mettre à jour l'état de la bande de LED ont été développées, de façon à permettre l'affichage des informations en provenance des capteurs de l'appartement connecté sur l'Ambient Atoms. Les requêtes POST sont construites dans OpenHAB en fonction des valeurs des capteurs.

\section{Travail à venir et conclusion}

Cet article introduit l'Ambient Atoms, un nouvel objet connecté pour la visualisation ambiante. Ce périphérique est générique, et peut donc être adapté pour des applications diverses. Un premier prototype a été développé pour une application de visualisation ambiante de diverses informations relatives à un appartement intelligent. Ce premier prototype à permis d'intégrer l'Ambient Atoms dans un système de domotique exitant. Il a aussi permis de premiers test encourageants. La suite des travaux visera à réaliser une évaluation plus formelle de l'Ambient Atoms sur un cas d'usage qui pourra être celui de l'appartement connecté, mais aussi sur d'autres cas. Lors de ces évaluations, différents modes de visualisation (couleurs/animations) pourront être testés dans différents contextes, afin de proposer des règles d'usage. Le prototype actuel intègre neuf atoms, mais ce nombre n'est pas lié à une limite technique. Des Ambient atoms de différentes tailles, et avec plus d'atoms pourront donc aussi être envisagés afin de définir, une éventuelle limite ergonomique à la quantité d'informations restituées. Ce premier prototype nous a permis de mieux appréhender les problématiques à approfondir. Parmi elles, une question importante est celle de rendre l'utilisateur acteur de la conception de son Ambient Atoms. Comment faciliter la personnalisation de la forme et du comportement du dispositif ? On pourra par exemple se demander si une solution basée sur IFTTT serait acceptable, ou si un langage de programmation spécifique serait plus adapté.

\section{Remerciements}

Ce projet a été conduit dans le cadre de l'Equipex Amiqual4Home [16] ANR-11-EQPX-0002. Les auteurs tiennent à remercier Nicolas Bonnefond et Rémi Pincent pour leur aide sur les outils de prototypage et sur les services OpenHAB [18], ainsi que James Crowley, directeur d'Amiqual4Home, pour avoir permis la réalisation de ce projet.

\section{Bibliographie}

[1] Z. Pousman and J. Stask, "A Taxonomy of Ambient Information Systems: Four Patterns of Design," in Proc. AVI'2006, 2006.

[2] S. Consolvo, P. Roessler and B. E. Shelton, "The CareNet Display: Lessons Learned from an In Home Evaluation of an Ambient Display,” in UbiComp 2004 - Ubiquitous Computing, 6th International Conference, 2004.

[3] T. Miller and J. Stasko, "The InfoCanvas: information conveyance through personalized, expressive art," in in Proc. of CHI'2001, 2001.

[4] J. Fogarty, J. Forlizzi and S. Hudson, "Aesthetic Information Collages: Generating Decorative Displays that Contain Information," in Proc. of the UIST 2001, 2001.

[5] "Chumby," [Online]. Available: http://www.chumby.com/.

[6] E. D. Mynatt, J. Rowan, A. Jacobs and S. Craighil, "Digital Family Portraits: Supporting Peace of Mind for Extended Family Members," in Proc. CHI 2001.

[7] S. Choi, H. Jeong, M. Ko and U. Lee, "LockDoll: Providing Ambient Feedback of Smartphone Usage within Social Interaction," in Proc. CHI 2016, 2016.

[8] M. Weiser and J. S. Brown, “Designing Calm Technology,” Xerox, 1995. 
[9] A. Chang, B. Resner and B. Koerner, "LumiTouch: An Emotional Communication Device," in Proc. CHI 2001, 2001.

[10] “Ambient umbrella," [Online]. Available: http://www.geeky-gadgets.com/the-ambient-umbrella-30-12-2009.

[11] A. Gustafsson and M. Gyllenswärd, "The Power-Aware Cord: Energy Awareness through Ambient Information Display," in Proc. CHI 2005, 2005.

[12] H. Evans and H. Hansen, “"Nuage Vert,"," 2008. [Online]. Available: http://hehe.org.free.fr/hehe/texte/nv/index.html.

[13] [Online]. Available: http://www.ambientdevices.com.

[14] M. Pesce, 2012. [Online]. Available: http://MooresCloud.com. [Accessed 2014]

[15] A. Dahley, C. Wisneski and H. Ishii, "Water Lamp and Pinwheels: Ambient Projection of Digital Information into Architectural Space,” in Proc. CHI 1998, 1998.

[16] “Amiqual4Home,” [Online]. Available: https://amiqual4home.inria.fr/home/.

[17] "particle.io," [Online]. Available: https://www.particle.io/.

[18] “OpenHAB," [Online]. Available: http://www.openhab.org/.

[19] S. Kirsner, "Peripherals, Lite-brite style," 2002. [Online]. Available: http://archive.wired.com/wired/archive/10.02/mustread_pr.html.

[20] A. Gyllenswärd and M. Gustafsson, "The power-aware cord: energy awareness through ambient information display," in Proc. ACM CHI'05 Extended Abstracts, 2005.

[21] J. Kim, Y. Kim and T. Nam, “The Ténéré: Design for Supporting Energy Conservation Behaviors,” in Proc. ACM CHI'09 Extended Abstracts, 2009.

[22] T. Holmes, "Eco-visualization: combining art and technology to reduce energy consumption," in Proc. ACM SIGCHI on Creativity \& Cognition, 2007. 